\title{
A new simple method for determining the sound absorption coefficient
}

\author{
Merab Chelidze* \\ Institute of Machine Mechanics, Mindeli st. 10, Tbilisi, Georgia.
}

\begin{abstract}
Taking into account the influence of the length and diameter of the impedance tube on the process of decay of sound waves, a new study of sound propagation in an impedance tube is presented, on the basis of which it is easier to determine the sound absorption coefficient. The new simple method of determining the absorption coefficient, based on the decay of reverberating waves, is fairly stable and demonstrates a low sensitivity for all errors made in the measurement. The presented method makes it possible to measure the sound absorption coefficient without laboratories and precision measuring instruments in a wide range at the level of separate consumers.
\end{abstract}

\section{Introduction}

More people than ever are now complaining about unwanted noise pollution - from rowdy neighbors and loud traffic to late-night pubs and clubs. The increasing demand for quiet in buildings and auditoriums has stimulated the use and development of materials with soundabsorbing qualities. Noise control can be achieved by reducing the intensity of sound to the level that is not harmful to a human ear. There are four basic principles employed to reduce noise which is absorption, isolation, vibration isolation, and vibration damping. In fact, the most recognized technique to reduce noise is sound absorption on the materials itself. Currently, one of the important topics in acoustic science is noise control. It is important to control the noise in order to minimize extraneous noise in the environment, buildings and our rooms [1].

The sound incident on a material is reflected, absorbed, and transmitted, as indicated in Figure 1. The reflected sound then proceeds to a second wall where it is again reflected, absorbed, and transmitted, and so on, experiencing many times reflections before its energy is all absorbed or transmitted [1,2].

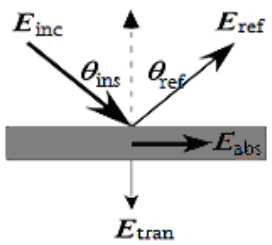

Figure 1. Decomposition of the incident sound energy $E_{i n c}$ on the $E_{r e f}, E_{a b s}$ and $E_{t r a n}$ energies

*Corresponding autor: merabchelidze@yahoo.com 
The absorption coefficient $\alpha$ is defined as the ratio of absorbed energy to incident energy

$$
\alpha=\frac{E_{a b s}}{E_{\text {ins }}}
$$

Sometimes $\alpha$ is defined as the ratio of all energy not reflected to incident energy, i.e. $\alpha=1-\mathrm{E}_{\mathrm{ref}} / \mathrm{E}_{\mathrm{ins}}=\left(\mathrm{E}_{\mathrm{abs}}+\mathrm{E}_{\text {tran }}\right) / \mathrm{E}_{\text {ins. }}$. By conservation of energy $E_{\text {ins }}=E_{\text {ref }}+E_{\text {abs }}+E_{\text {tran. }}$. The materials absorption coefficient is limited between 0 (not absorbent at all, i.e. reflective) and 1 (totally absorbent) [2,3].

It should be noted that in existing methods, the sound absorption coefficient is not directly determined by formula (1). There are various methods for obtaining the absorption coefficient, in which the results obtained differ significantly from one another $[3,4]$.

Besides of the vacuum, sound is propagated in any materials. Sound is appeared by any disturbance of the air pressure $p$; density $\rho$; environment particles speed $v$ and temperature $T$. The functional connection between air pressure and density is expressed by adiabatic equation $p=f(\rho)$. The differentiation of which gives the sound composition equation $[5,6]$.

$$
\frac{\partial \rho}{\partial t}=\frac{1}{c^{2}} \frac{\partial p}{\partial t}
$$

where $c$-is the speed of sound propagation and $t$ is the time.

Taking into account the Euler equations and the continuity of the flow, we obtain a onedimensional wave equation [5].

$$
\frac{\partial^{2} p}{\partial x^{2}}=\frac{1}{c^{2}} \frac{\partial^{2} p}{\partial t^{2}}
$$

where $x$ - coordinate on direction of the sound propagation

The solution of equation (3), for harmonic waves, takes the following view

$$
p=A \cos (\omega t-k x)+B \cos (\omega t+k x)
$$

where $A$ and $B$ - constants, $k=c / \omega$ - wave number, $\omega=2 \pi f$ - circular frequency, $f$ - number of wave oscillation per (on one) second.

In accordance with Eq. (4) the incident and reflected waves are summed i.e. depending on their phase they are increased and decreased.

Due to several reasons of complexity and capriciosity of existing measurement methods, one can find absorption coefficient values greater than one. Quite often engineers tend to round down to 1 any absorption coefficient result higher than 1 in order to represent a physical reality. However, values lower but close to unity remain untouched [3]. To the present day, the reverberation room (or random incidence) method is the most commonly used method for determining the absorption coefficient of a material.

The impedance tube is shown in Figure 2. For establishing plane waves the standards recommend a tube length of at least three diameters, but sometimes a length of at least 10 15 diameters is preferred. Plane waves in such tubes oscillate in the longitudinal direction along the length of the tube and cause oscillations of standing waves which can be fixed by a microphone in the form of oscillograms of the decay amplitudes.

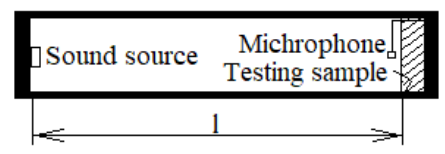

Figure 2. Impedance Tube 


\section{Research experiments}

Performed theoretical and experimental studies, with the help of modern digital technologies, have shown that the propagation of the waves of white noise in a closed tube creates and establishes a complex unstable reverberating oscillations (Figure 3a). It should be noted that longitudinal reverberating sound oscillations with the frequency of $f_{l}=300 \mathrm{~Hz}$ are clearly visible along the tube between the lids (stop-ends), whose frequency is equal to the ratio of the speed of sound $c$ to the double length of the tube $2 l$, i.e. $f_{l}=c / 2 l$ [7]. If the sound source is coherent, for example at a frequency of $2000 \mathrm{~Hz}$, coherent waves with a frequency of $2000 \mathrm{~Hz}$ are also installed in the tube, but in this case, little noticeable longitudinal oscillations with frequency $f_{l}=300 \mathrm{~Hz}$ are detected simultaneously (Figure 3b).

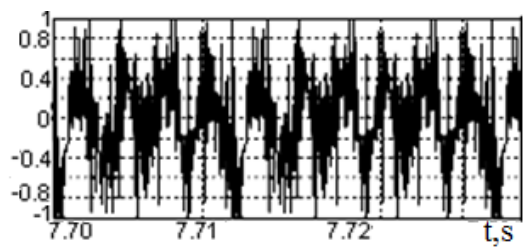

a) $\mathrm{f}_{1}=300 \mathrm{~Hz} ; \tau=0.0033 \mathrm{sec}$.

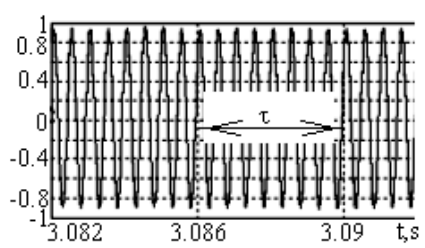

b) $\mathrm{f}=2000 \mathrm{~Hz} ; \tau_{\mathrm{f}}=0.0005 \mathrm{sec} ; \tau=0.0036 \mathrm{sec}$.

Figure 3 Reverberating white a) and coherent $b$ ) sound waves in the impedance tube

In the tube, from the moment the sound source is turned off, a transient process of decay of the reverberating amplitudes occurs until the sound waves completely disappear (Figure 4). The frequency of the decay of longitudinal waves with white noise is $\mathrm{f}_{1}=300 \mathrm{~Hz}$, and for a coherent noise source it is $\mathrm{f}_{\mathrm{l}}=275 \mathrm{~Hz}(\tau=0.0036) \mathrm{sec}$, and both are related to the length of the impedance tube.

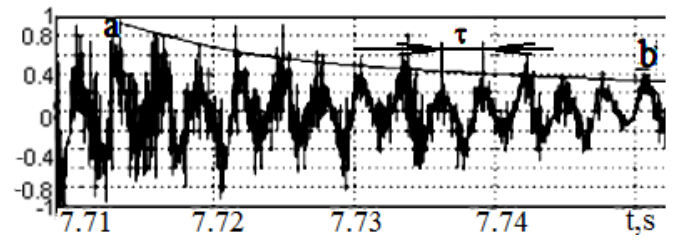

a) $\mathrm{f}_{1}=300 \mathrm{~Hz}, \tau=0.0033 \mathrm{sec}$.

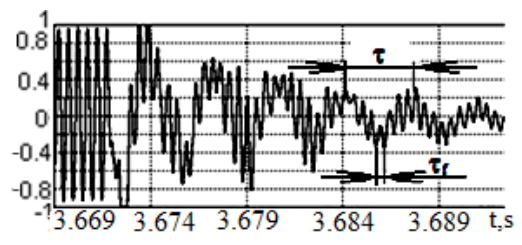

b) $\mathrm{f}_{1}=275 \mathrm{~Hz} ; \tau_{\mathrm{f}}=0.0005 \mathrm{sec}$. $\mathrm{f}=2000 \mathrm{~Hz}$

Figure 4. Decay processes of white a) and coherent b) reverberating sound waves.

According to Figure $4 \mathrm{~b}$, the period $\tau$ and the frequency $f$ of the decay reverberant sound amplitudes can be calculated as follows. The period of coherent sound waves $\tau_{f}$ is equal to subtracting time mark numbers divided by the number of amplitudes of this interval $\tau_{f}=(3.689-3.684) / 10=0.0005 \mathrm{sec}$. Thus, in accordance with the period $\tau_{\mathrm{f}}$, the frequency of the noise source is $f=1 / \tau_{f}=1 / 0.0005=2000 \mathrm{~Hz}$. Consequently, the period and frequency of longitudinal oscillations will be $\tau=n \tau_{f}=8^{*} 0.0005=0.004 \mathrm{sec}$ and $f=1 / \tau=250 \mathrm{~Hz}$. Where $n$ is the number of amplitudes in the period $\tau$. The curve "ab" in Figure 4a shows the average rate of the decay process of amplitudes of reverberating longitudinal waves.

Figure 5 shows the decay processes of coherent sound sources corresponding to frequencies $100,200,500$ and $1000 \mathrm{~Hz}$ in an impedance tube with a length $l=0.66 \mathrm{~m}$.

As can be seen from the oscillogram in all four cases, the amplitude decay processes occur in one and the same frequency of longitudinal oscillations $f_{l}=275 \div 280 \mathrm{~Hz}$. The decay 
frequencies of these oscillograms are far from the original frequencies of the sound source, except for the oscillogram shown in Figure $4 \mathrm{~b}$, where the attenuation frequency $f_{l}=275 \mathrm{~Hz}$ is close to the initial frequency of the sound source $f=200 \mathrm{~Hz}$. Proceeding from the obtained oscillograms of the decay of sound waves, it can be said that by changing the length and diameter of the impedance tube, a condition can be reached in which the frequencies of the established and decay regimes are equal, which makes it possible to considerably simplify the determination of the sound absorption coefficient.

a)

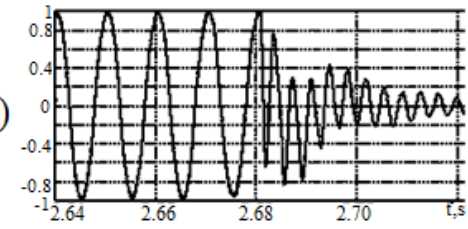

c)

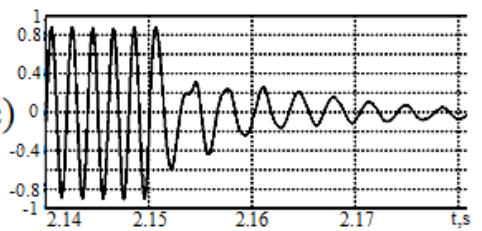

b)

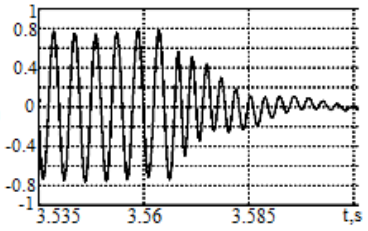

d)

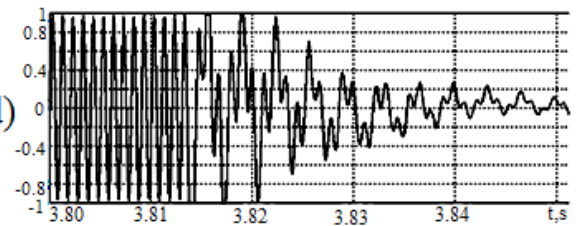

Figure 5. Sound decay processes of coherent noise frequencies of 100, 200, 500 and $1000 \mathrm{~Hz}$.

Figure 6 shows the decay processes of reverberant sound amplitudes at attenuation frequencies equal to the frequencies of the sound sources. To achieve this, the length of the tube should be $1 / 4$ of the length of the sound waves. In addition, to ensure plane waves, the diameter of the tube should be less than $1 / 3$ of the length of the tube. It should be noted that the oscillograms of 5000 and $10,000 \mathrm{~Hz}$ are distorted, especially the $10,000 \mathrm{~Hz}$ oscillograms since they are obtained by the tube length of $l=0.025 \mathrm{~m}$. On this basis, in the longitudinal frequency period $\tau=0.003 s(3300 \mathrm{~Hz}), 3$ amplitudes of the noise source of $10,000 \mathrm{~Hz}$ are observed. For a smaller tube length $(20 \div 8 \mathrm{~mm})$ and diameter (about of 5 $\mathrm{mm}$ ), a microphone used in laptops and smartphones was needed.
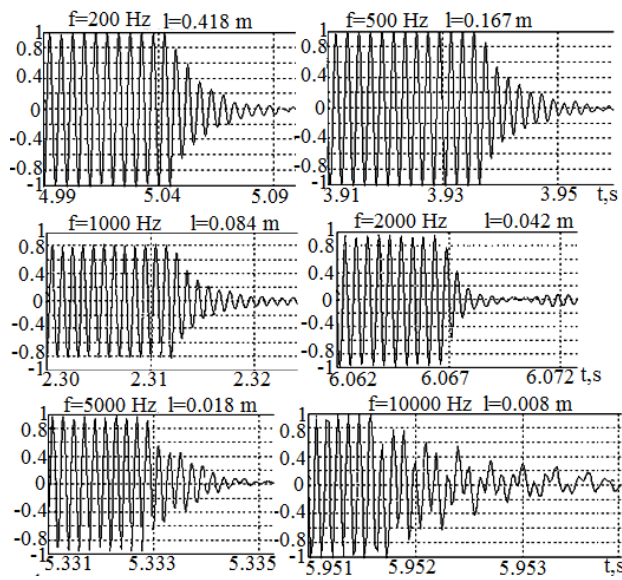

Figure 6. Achievement of equality of coherent sound sources and decay frequencies by means of regulation of the tube length.
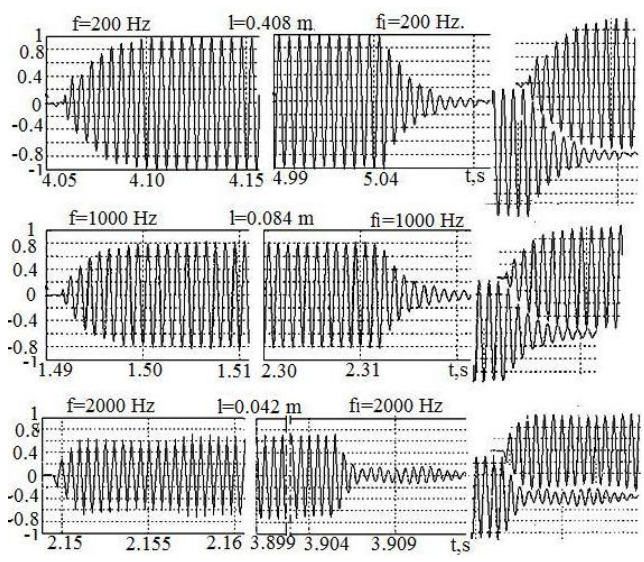

Figure 7. Mirror images of forming and decay of reverberating coherent sound amplitudes in a closed space 
With a low-power sound source during the study, it was also found that in closed chambers (in the tube), the growth and decay of the sound amplitudes occurs at the same rate and they have the form of mirror images (Figure 7).

Especially it should be noted that the process of destruction of the reverberating sound amplitudes obtained by the method described above is very stable. In addition, the accuracy of the measurement is practically independent of the measurement conditions, types of measuring instruments and their accuracy.

According to the oscillograms of the decay of reverberating sound waves, the sound absorption coefficient can be calculated from the following simple formula (5), analogously as occurs with calculating the damping coefficient in the theory of oscillations $[7,8]$,

$$
\alpha_{k}=\frac{A_{a}-A_{b}}{m A_{a}}
$$

where $A_{a^{-}}$and $A_{b}$ - are the maximum and minimum amplitudes of the ab-interval, $\mathrm{m}$ - is the number of amplitudes of the interval "ab" (Figure 4a). The nonlinear curve "ab" by formula (5) is assumed to be linear.

In the theory of oscillations, when the average value is determined approximately, the absorption coefficient for the entire nonlinear interval is calculated taking into account in formula (5) the coefficient 2 [9].

$$
\alpha_{k}=\frac{2}{m} \frac{A_{a}-A_{b}}{A_{a}}
$$

Based on numerous experimental studies, a new method for measuring the sound absorption coefficient showed a greater sensitivity to the measurement of the acoustic properties of materials. Figure 8 shows some oscillograms of the decay of the sound amplitudes, the absorption coefficients of which were calculated from formula (5). In addition, the method for determining the sound absorption coefficient, based on the decay of the sound amplitudes, proved to be very stable when the measurement requirements were violated. For example, Figure 9 shows the entering and decay waveforms that were obtained for a wet porcelain plate when, in one case, the tube cover was tightly closed $\alpha_{\kappa}=0.125$ and in another case, the cover on the side of the noise source was practically open $\alpha_{\kappa}=0.128$. Since the absorption coefficient is determined by the ratio of the subtraction of the maximum and minimum amplitudes to the maximum amplitude of the given interval, the errors appearing due to inaccuracies of the measuring devices, instability of measurement conditions and the environment are not large, unlike in the existing methods in which they greatly affect the obtained results.
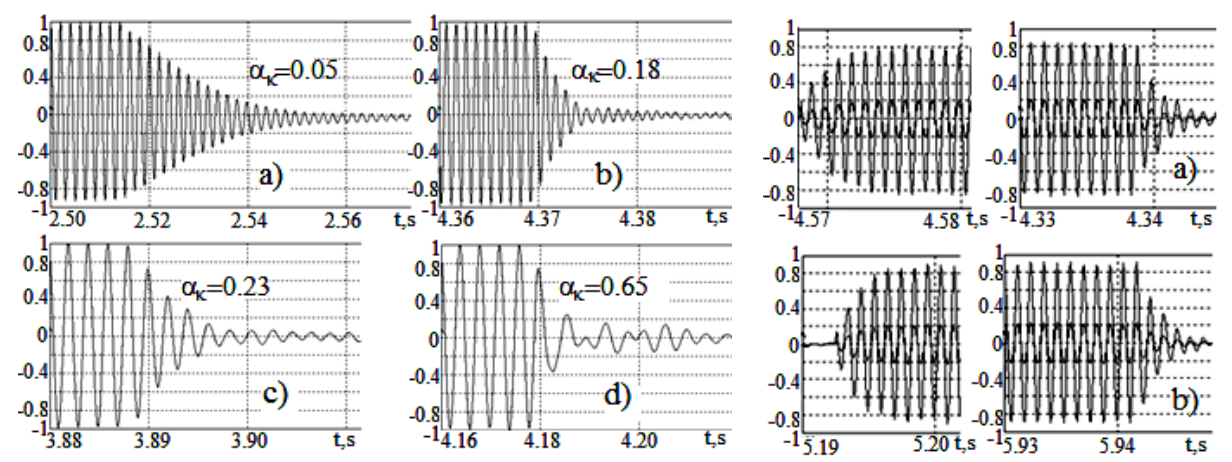

Figure 8. Oscillograms of attenuation amplitudes at 500 Hz with samples of a) water, b) tiles, c) plywood and d) cotton.

Figure 9. Oscillograms a) a tightly closed lid, b) an incomplete lid on the side of the noise source. 
If equal values appear in the numerator and denominator simultaneously, than in our approach the measurement errors will will not cause the change of the fraction. Here the main thing is that during the decay of the sound amplitude (approximately $0.01 \mathrm{sec}$ ), no changes occurred on the background sound.

\section{Conclusion}

The conducted studies showed that, according to the proposed method, the results of the test for the absorption coefficient do not depend on the condition of performing test, that is, the absorption coefficients of the same material with the same properties do not depend on the test condition, accuracy, and type of measuring instruments. The method of determination of the absorption coefficient by means of decay of the reverberating waves is sufficiently stable, demonstrates a high sensitivity to the materials acoustic characteristics and a low sensitivity to errors admitted during the measurement. Therefore, it can be concluded that, after some further improvement, this method has a good prospect of widespread use in the individual determination of the absorption coefficient of materials.

\section{References}

[1] F.R. Watson. The Absorption of Sound by Materials. University of Illinois Bulletin. (2007)

[2] J. Kunioa. A comparison of two and four microphone standing wave tube. (2009)

[3] R.T. Muehleisen. Measurement of the Acoustic Properties of Acoustic Absorbers. (2011)

[4] M. McGrory, D.C. Cirac, O. Gaussen, D. Cabrera. Sound absorption coefficient measurement: Re-examining the relationship between impedance tube and reverberant room methods (1) 21-23 November (2012)

[5] E. Skudrzyk. The Foundations of Acoustics. Basic Mathematics and Basic Acoustics. SpringerVerlag, New York, (1971)

[6] D. R. Raichel. The science and applications of acoustics. The City University of New York. Springer, (2012)

[7] M.A. Chelidze, D. Nizharadze, J. Javaxishvili, M. Tedoshvili. Investigation of Sound Absorption. Modern achivements of Science and Education VIII International Conference September 29 October 06, Jerusalem (Israel) pp. 66-71. (2016)

[8] M.A. Chelidze. Investigation of Sound Absorption Coefficient by Decay of Standing Waives in The Impedance Tube. GESJ: Physics 2017 | No.1(17) ISSN 1512-1461.

[9] M.A. Chelidze, J. Javaxishvili, D. Nizharadze, M. Tedoshvili. Investigation of Sound Absorption coefficient by Energetic Method. Proceedings of VII International Conference on Science and Education. April 27-May4, Rome (Italy) pp 3-9. (2017) 